\title{
Applications of Genetic Algorithm on Optimal Sequence for Parrondo Games
}

\author{
Degang Wu and Kwok Yip Szeto \\ Department of Physics, The Hong Kong University of Science and Technology, Clear Water Bay, Hong Kong, Hong Kong \\ wuab@connect.ust.hk,phszeto@ust.hk
}

Keywords: Genetic Algorithm, Parrondo Game, Optimization, Game Theory.

Abstract: $\quad$ Parrondo game, which introduction is inspired by the flashing Brownian ratchet, presents an apparently paradoxical situation where there are ways to combine two losing games into a winning one. The original Parrondo game consists of two individual games, game A and game B. Game A is a slightly losing coin-tossing game. Game B has two coins, with an integer parameter $M$. If the current cumulative capital (in discrete unit) is a multiple of $M$, an unfavorable coin $p_{b}$ is used, otherwise a favorable $p_{g}$ coin is used. Game B is also a losing game if played alone. Paradoxically, combination of game A and game B could lead to a winning game, either through random mixture, or deterministic switching. In deterministic switching, one plays according to a sequence such as ABABB. Exhaustive search and backward induction have been applied to the search for optimal finite game sequence. In this paper, we apply genetic algorithm (GA) to search for optimal game sequences with a given length $N$ for large $N$. Based on results obtained through a problem-independent GA, we adapt the point mutation operator and one-point crossover operator to exploit the structure of the optimal game sequences. We show by numerical results the adapted problem-dependent GA has great improvement in performance.

\section{INTRODUCTION}

Inspired by the flashing ratchet, Parrondo invented the games of chance later known as Parrondo game (Parrondo, 1996), in which two losing games can be combined following a random or periodic strategy leading to a winning game. Later, (Allison and Abbott, 2002) and (Toral et al., 2003) demonstrated that Parrondo game can be described by a discrete Fokker-Planck equation, thus a more rigorous relation between Parrondo game and Brownian ratchet was established. From the perspective of game, the optimal sequence for a given set of parameters for Parrondo games was inferred by Dinis using dynamic programming (Dinis, 2008). It is worth noting that greedy algorithms do not work in finding the optimal game sequence for a finite length.

Multi-player versions of the games have been proposed, and they exhibit counterintuitive phenomena resembling those observed in game, control, and optimization theories or economics. Many researchers have found that greedy algorithms or strategies may lead to suboptimal or even losing solutions in these models (Dinis and Parrondo, 2003; Dins and Parrondo, 2004; Parrondo et al., 2007). Optimiza- tion problems of this type warrant the use of metaheuristic algorithm such as genetic algorithms (Goldberg and Holland, 1988; Holland, 1975).

The successful application of genetic algorithm has been demonstrated in the solution of many engineering problems, such as speed control of brushless DC motor (Xia et al., 2004), cyclic-steam oil production optimization problem (Patel et al., 2005), traveling salesman problem (Jiang et al., 2000), mobile robot motion control (Messom, 2002), modeling adaptive agents in stock markets (Fong and Szeto, 2001; Szeto and Fong, 2000), and the problem of airport scheduling (Shiu and Szeto, 2008). In science, genetic algorithm has also been used with good results in fields such as biology (Clote, 2005; Ding and Zhang, 2008; Pond et al., 2006), clusters (Doye, 2002; Wales et al., 2000; Wales et al., 1998) and glass transition (Debenedetti and Stillinger, 2001) in condensed matter physics.

Our objective of this paper is to use genetic algorithm on the search for optimal game sequence of long length. We first use Dinis algorithm to gain insight of the structure of optimal sequences with moderate length. We then propose structure-preserving evolution operators (mutation and one-point crossover 
operators), coupled with an appropriate structurepreserving population initialization procedure to find the optimal sequence for longer game sequence. Here structure-preserving condition means that the search process impose certain constraints on the patterns that are observed in the optimal sequences of moderate length, as found either by exhaustive search or Dinis algorithm. In order to assess the effect of the constraints on the quality of the solution, we first use simple genetic algorithm as a biased sampling method in order to justify the adaptive approach we use later. Our paper is organized as follows. In Section 2, we introduce the Parrondo game and discuss the Dinis algorithm that reveals some specific patterns which appear in the optimal sequence of moderate length. In Section 3, we propose structure-preserving mutation and one-point crossover and corresponding structure-preserving population initialization procedure. In Sections 4 and 5, we discuss the experiment design and present the experiment results. Finally, the concluding remarks can be found in Section 6.

\section{PARRONDO GAMES}

The original Parrondo game consists of two individual coin tossing games, namely game $\mathrm{A}$ and game $\mathrm{B}$. Game A has only one coin, whose winning probability is $p_{A}=1 / 2-\varepsilon$, where $\varepsilon$ is a small and positive number. Let $X(t)$ be the cumulative capital of the player at time $t$, an integer. If the player keeps playing game $\mathrm{A}$, the average capital satisfies

$$
\langle X(t+1)\rangle=\langle X(t)\rangle+2 p_{A}+1
$$

where $\langle\cdot\rangle$ is understood as ensemble average. Game B has two coins, one "good" coin and one "bad" coin. Game B has an integer parameter $M$. If $X(t)$ is a multiple of $M$, then $X(t+1)$ is determined by the "bad" coin with winning probability $p_{b}=1 / 10-\varepsilon$, otherwise the "good" coin with winning probability $p_{g}=3 / 4-\varepsilon$ is used. Similar to game A, if the player keeps playing game B only, the average capital satisfies

$$
\langle X(t+1)\rangle=\langle X(t)\rangle+2\left[\pi_{0}(t) p_{b}+\left(1-\pi_{0}(t)\right) p_{g}\right]-1
$$

which explicitly depends on $\pi_{0}(t)$, the probability that the capital $X(t)=0 \bmod M$. (Harmer and Abbott, 1999) showed that game $B$ is a losing game when $p_{b}=1 / 10-\varepsilon, p_{g}=3 / 4-\varepsilon$ and $M=3$, with positive $\varepsilon$. In this paper, we only discuss the case when $M=3$. There is a recent article discussing the phase diagram of the more complex situation where the games are two B games with different $M$ (Wu and Szeto, 2014).
Such extended Parrondo game with multiple $M$ exhibits interesting phenomena such as weak and strong Parrondo effects when the sequence is infinite.

If we model the Parrondo game as a discretetime Markov chain as in (Harmer and Abbott, $2002)$, we can define the probability vector $\pi(t) \equiv$ $\left(\pi_{0}(t), \pi_{1}(t), \pi_{2}(t)\right)^{T}$ (for $M=3$ ). Accordingly, the transition matrix for game $\mathrm{A}$ is

$$
\Pi_{A}=\left[\begin{array}{ccc}
0 & 1-p_{A} & p_{A} \\
p_{A} & 0 & 1-p_{A} \\
1-p_{A} & p_{A} & 0
\end{array}\right]
$$

such that the time evolution equation is $\pi(t+1)=$ $\Pi_{A} \boldsymbol{\pi}(t)$. Similarly, the transition matrix for game B is

$$
\Pi_{B}=\left[\begin{array}{ccc}
0 & 1-p_{g} & p_{g} \\
p_{b} & 0 & 1-p_{g} \\
1-p_{b} & p_{g} & 0
\end{array}\right]
$$

Parrondo game can be played according to a deterministic finite game sequence such as $\mathrm{ABABB}$, so at the end of the sequence, the probability vector $\pi(5)$ is

$$
\boldsymbol{\pi}(5)=\Pi_{B} \Pi_{B} \Pi_{A} \Pi_{B} \Pi_{A} \pi(0) .
$$

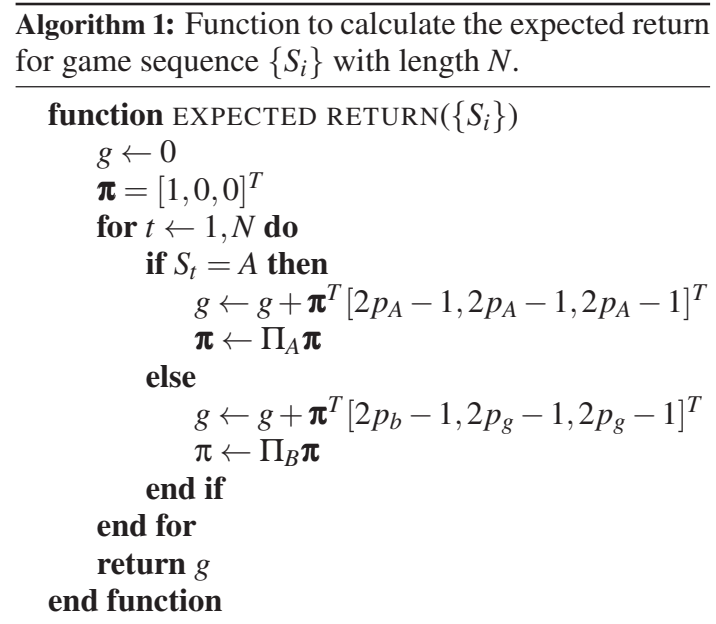

Parrondo game has a seemingly paradoxical property that while game A and B are losing when they are played individually, the stochastic mixture of game $\mathrm{A}$ and $\mathrm{B}$, or playing according to a deterministic sequence may lead to a winning combined game for small positive value of $\varepsilon$. For the detailed analysis of this paradox, please refer to (Harmer and Abbott, 2002). For a finite game sequence with length $N$, the expected return at the end of the game sequence can be computed by Algorithm 1. Our task is to find the finite game sequence that has maximum cumulative gain. The expected return per game in the stationary state for a periodic sequence with length $N$ 
can be computed using Algorithm 1 with two minor modifications: the initial value of $\boldsymbol{\pi}$ should be the equilibrium distribution of the transition matrix $\Pi_{\alpha(1)} \Pi_{\alpha(2)} \cdots \Pi_{\alpha(N)}$ and the final value of $g$ should be divided by $N$.

Sequences up to period 12 have been studied using symbolic manipulators and exhaustive search (Wagon and Velleman, 2001), and the periodic sequence $\mathrm{ABABB}$, or any of its permutations, has come up as the best in the sense that it provides the highest returns in the stationary state. However, exhaustive search for optimal sequences of finite length $N$ is not feasible for large $N$. Using dynamic programming, Dinis discovered that optimal sequences with finite $N$ "consist of several repetitions of the ABABB motif flanked by brief pieces of other sequences. (Dinis, 2008) For example, the optimal sequence for $N=20$ with initial condition $X(t)=0$ and $\varepsilon=0$ is AB ABABBABABBABABB ABB. In fact, the structure of the optimal sequences is more specific: they are strings of $\mathrm{AB}$ and $\mathrm{ABB}$. From these results, we have the following definition:

Definition 1. A game sequences is said to have a special structural-property if it is made of $A B$ and/or $A B B$ substrings exclusively.

Straightforward implementation of Dinis algorithm requires storage space that scales as $N$, in order to store all the numerical results from all intermediate steps. Moreover, the algorithm approximates two-dimensional plane with a discrete square grid (In (Dinis, 2008), a $2000 \times 2000$ grid was used for each step). The error in the expected payoff due to grid approximation will accumulate across time and the total error in the expected payoff at the end of the sequence is at least a linear function of $N$. The effect of this error to the correctness of the optimal sequence has not been investigated. The approximation therefore constraints the computation of optimal sequences for large $N$. This suggests that heuristic algorithms such as GA that demands much less space and does not require approximation in the expected payoff are useful for optimal sequences with long length. Moreover, by definition, Dinis' algorithm does not provide any information of the suboptimal sequence. In contrast, while searching for the optimal solution, GA also efficiently samples many suboptimal solutions, which provide insights in the relation between the structure of a sequence and its performance.

\section{STRUCTURE-PRESERVING GENETIC ALGORITHM}

\subsection{Representation}

If we map game $\mathrm{A}$ to 0 and map game $\mathrm{B}$ to 1 , a game sequence with length $N$ can be mapped to a binary string with the same length, and the order of the binary values corresponds to the order the games are played. The objective function is given by Algorithm 1. For example, the game sequence $\mathrm{ABABB}$ is encoded as 01011 . For game sequences that are strings of $\mathrm{AB}$ and $\mathrm{ABB}$, an auxiliary representation is useful: 0 for $A B$ and 1 for $A B B$. For example, game sequence $\mathrm{ABABB}$ is encoded as 01 in this auxiliary representation. However, game sequences under this auxiliary representation vary in length from sequence to sequence. To distinguish these two kinds of representations, we call the one that maps game A to 0 the original representation.

\subsection{Simple Genetic Algorithm}

A simple genetic algorithm is used as the reference.

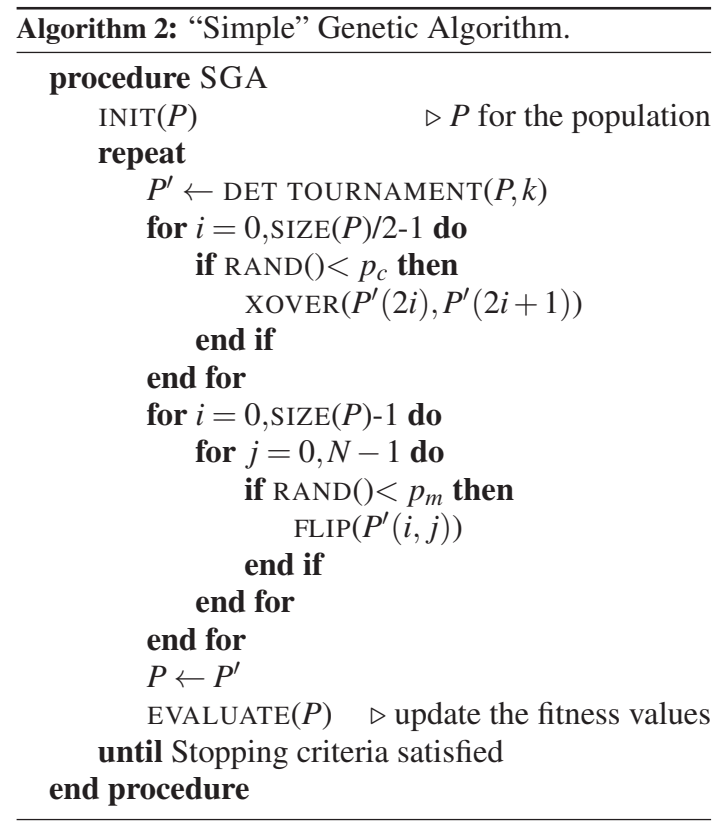

In the initialization, each locus of each chromosome is set to 1 or 0 with equal probability. The new population $P^{\prime}$ is generated from the last one using deterministic tournament: from previous generation, randomly draw $k$ chromosomes with equal probability, and select the one with the highest fitness value. 
This process is repeated until the new population has the same number of chromosomes as the previous population. The one-point crossover operator is denoted by XOVER(), and its rate is denoted by $p_{c}$. The mutation rate per bit is denoted by $p_{m}$.

\subsection{Structure-preserving Mutation Operator}

If a chromosome has special structural property, we can apply a mutation operator that preserves this property. We propose a structure-preserving mutation operator in Algorithm 3.

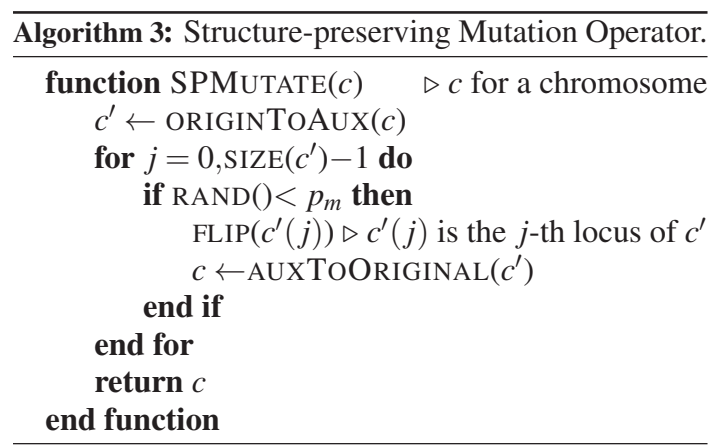

However, when we use Algorithm 3, we must check if the sequence after mutation has length $N$. We therefore need an algorithm for chromosome repair, using first a conversion function AUXTOORIG$\operatorname{INAL}\left(c^{\prime}\right)$. This function converts $c^{\prime}$ in the auxiliary representation back to a chromosome in the original representation, which is not always possible because the converted chromosome in original form may have a length greater or less than $N$. If $\operatorname{SIZE}(c)>N$, we then pop 01 or 011 from the back of $c$ until $\operatorname{SIZE}(c)<N$. We append 01 or 011 to the back of $c$ such that $\operatorname{SIZE}(c)=N$.

\subsection{Structure-preserving Crossover Operator}

If a chromosome has special structural property, we can apply an adapted one-point crossover operator that preserves this propose. The structure-preserving one-point crossover operator is shown in Algorithm 4.

Here the function CHOOSEXPT decides the crossover point in the auxiliary representation such that the two children chromosomes in the original representation are either one bit longer or shorter than $\mathrm{N}$. The function FIXCHROMOSOME lengthens or shortens the chromosome by mutating 01 to 011,011 to 01 , converting 011 to 0101 , or converting 0101 to 011 .

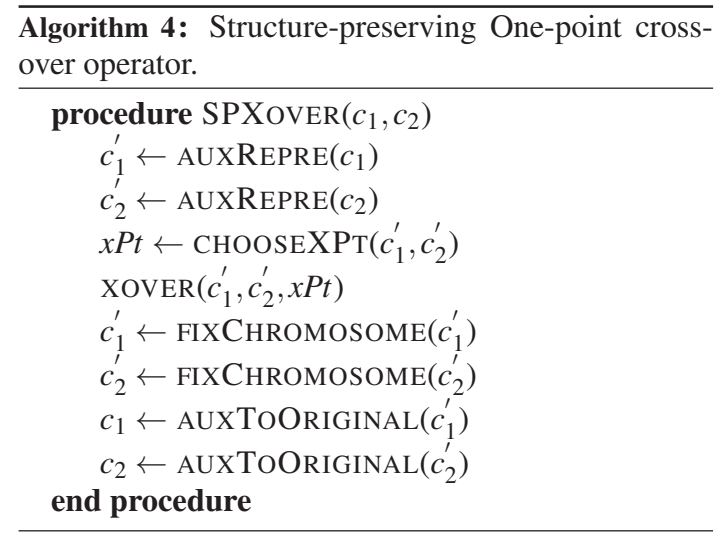

\subsection{Structure-preserving Genetic Algorithm}

In order to replace the mutation operator and onepoint crossover operator with the structure-preserving versions, the chromosomes must have special structural property at all time. For this purpose, a structurepreserving initialization routine is propose in Algorithm 5 .

As we will show later, this initialization routine per se greatly improves the quality of the initial chromosomes. In this paper, the structure-preserving genetic algorithm (SPGA) refers to ones that run in the framework of Algorithm 2, with population initialized by Algorithm 5, the mutation operator replaced by Algorithm 3 and the one-point crossover operator replaced by Algorithm 4. This combination of algorithms in SPGA reduces the search space substantially. One concern about this reduction is that it might fragment the solution space into disjoint subspace. We will discuss this issue in Section 5.

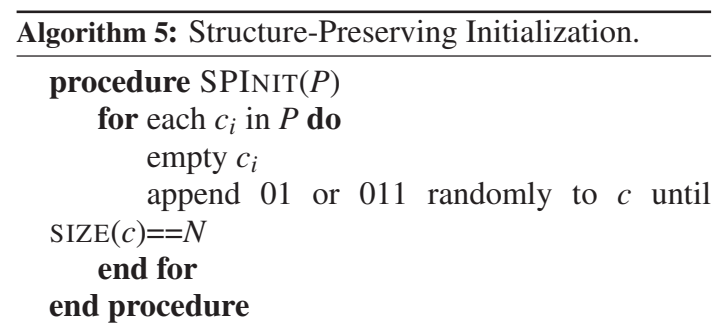

\section{EXPERIMENTAL DESIGN}

For comparison of the relative efficiency of the various components of our genetic algorithm, we consider the following versions of GAs. 
- SGANoX: A GA that uses only the mutation operator, with uniform initialization and no crossover operators

- SGANoXwSPInit: SGANoX with structurepreserving initialization

- SGA: Simple Genetic Algorithm, with uniform initialization, standard mutation operator and onepoint crossover operator

- SGAwSPInit: Simple Genetic Algorithm with structure-preserving initialization, standard mutation operator and one-point crossover operator

- SPGANoX: Structure-preserving mutation only genetic algorithm with structure-preserving initialization and structure-preserving mutation operator, but without crossover

- SPGA: Structure-preserving genetic algorithm with structure-preserving initialization, structurepreserving mutation operator and structurepreserving crossover operator

For simplicity, when we use the term SGAs, we mean the group of SGA, including SGANoX, SGA, SGANoXwSPInit, SGAwSPInit. Similarly, when we use the term SPGAs, we mean the group of SPGA, including SPGANoX, SPGA.

The size of the population, $N_{P}$, will be set to 100 for all versions of GAs. The size of the tournament, $k$, defined in Algorithm 2 in the deterministic tournament selection process will be set to 10 . The crossover rate, $p_{c}$ and the mutation rate per bit, $p_{m}$, both defined in Algorithm 2, will be chosen individually for each GA such that each GA has good performance.

\section{RESULTS AND ANALYSIS}

First we investigate the effect of imposing structurepreserving evolution operators and population initialization on search space. Exhaustive search is not an option for sequences, as long as $N=80$. Instead we use SGA to perform a biased sampling on the search space by just running SGA with $p_{m}=0.02$ and $p_{c}=1.0$. The selection mechanism in SGA biases towards chromosomes having higher fitness values. Every chromosome that appears during 200 generations of evolution from 50 independent experiments are collected as samples. Numerical results show that the fitness distribution for game sequences satisfying special structural property are strongly grouped together at high fitness values, and they are among the chromosomes having fitness values mostly higher

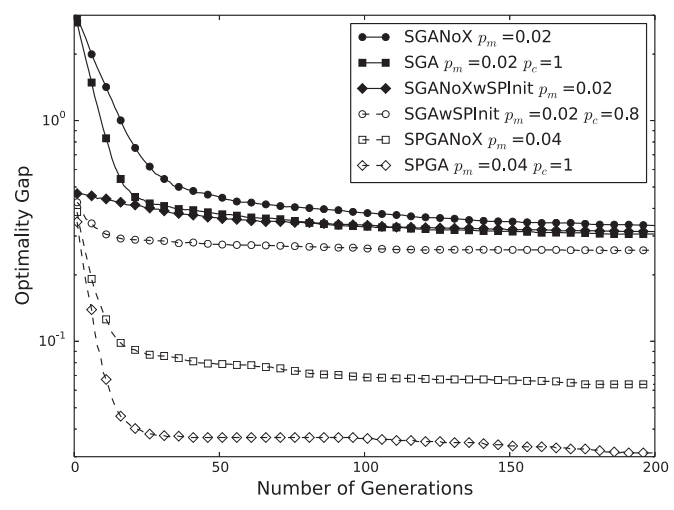

Figure 1: Optimality gap as a function of number of generations for $N=80$. Every curve is an average from 100 independent experiments. Y-axis is in log-scale. The gap is smallest for SPGA, indicating it is the best performing GA.

than the highest fitness of the sequences without special structure. From this observation, we conclude that imposing structure-preserving evolution operators and population initialization on search space will not fragment the search space. Furthermore, the usage of structure-preserving evolution operators and population initialization can be justified due to the overall higher fitness value obtained.

Next we compare the performances of the various GAs mentioned in the last section on the search for optimal sequence of with $N=80$. We first calculate the true expected return of the optimal sequence using Dinis' algorithm. We then measure the performance of various GAs by the optimality gap, the difference between the expected return of the optimal sequence and the expected return of the chromosome with the highest fitness value. We show the optimality gap as a function of number of generations in Fig. 1. The values of $p_{m}$ and $p_{c}$ are chosen on the basis of performance.

The structure-preserving initialization alone contributes significantly to the performance, as can be seen from the relative performances of SGANoX and SGA with or without structure-preserving population initialization in Fig. 1. In the case of problem-independent GAs, one-point crossover operator contributes to the early edge in performance. The problem-dependent structure-preserving GAs generally perform better. SPGANoX outperforms SGAwSPInit. SPGA performs the best.

Since there is no guarantee that optimal game sequence will be obtained after 200 generations, we therefore need to assess the average quality of the best solutions after 200 generations. For $\mathrm{N}=80$, the optimal game sequence is

ABXXXXXXXXXXXXXXXABB, 
where $\mathrm{X}$ stands for $\mathrm{ABABB}$. We call those $\mathrm{AB}$ (or $\mathrm{ABB}$ ) substring that are not able to form $\mathrm{ABABB}$ with adjacent substring ungrouped $\mathrm{AB}$ (or $\mathrm{ABB}$ ).

A typical suboptimal but still good solution resulted from SPGAs has the following structure:

XXXXXXАВBXXXXАВBXXXАВXАВ,

which possesses the right number of $\mathrm{AB}$ and $\mathrm{ABB}$ substrings, and the SPGAs manage to figure out the overall structure. From this observation, we expect that a problem-specific local search can obtain the real optimal solution from the best solution obtained from SPGAs. In this context, we may introduce some alternative quality measures by the following enquiries:

1. Has the right number of $\mathrm{AB}$ and $\mathrm{ABB}$ been reached? (Here the right number refers to the number in the optimal sequence.) If not, calculate the deviation by

$$
d_{1}(s)=\left|N_{A B}(s)-N_{A B}\left(s^{*}\right)\right|+\left|N_{A B B}(s)-N_{A B B}\left(s^{*}\right)\right|,
$$

where $N_{A B}(s)\left(N_{A B B}(s)\right)$ is the number of $\mathrm{AB}$ (ABB respectively) and $s^{*}$ is the optimal sequence.

2. If the right number of $\mathrm{AB}$ and $\mathrm{ABB}$ has been reached, has the right number of ungrouped $\mathrm{AB}$ and $\mathrm{ABB}$ been reached? (Here, the right number again refers to the number in the optimal sequence.) If $N_{A B}^{*}(s)\left(N_{A B B}^{*}(s)\right)$ is the number of ungrouped $\mathrm{AB}$ ( $\mathrm{ABB}$ respectively) and $s^{*}$ is the optimal sequence, then this deviation can be written as

$$
d_{2}(s)=\left|N_{A B}^{*}\left(s^{*}\right)-N_{A B}^{*}(s)\right|+\left|N_{A B B}^{*}\left(s^{*}\right)-N_{A B B}^{*}(s)\right| .
$$

For both measures, the smaller the values of $d_{1}(s)$ and $d_{2}(s)$ the higher is the quality of the sequence. We show the statistics of the best chromosomes obtained by various GAs at the end of 200-th generation in Table 1. Data are collected from 100 independent experiments for each GA variant. As can be seen from the table, SPGAs are better at figuring out the structure of the optimal sequence than other GAs.

We see that for medium size sequence $(N=80)$, our SPGA is good approximation to Dinis analysis, as shown in Fig. 1. Thus for medium size sequence, we do not see the advantage of our SPGA algorithm.

We now apply the same set of GAs on searching for the optimal sequences with longer length $N$. The values of $p_{m}$ and $p_{c}$ are the same as those used before. Note that the exact optimal sequence with length $N=200$ is not easily available as straightforward implementation of Dinis algorithm needs huge amount of storage space and exhaustive search will require even more. Thus, we use an alternative measure of performance: we record the highest fitness value in the population as a function of the number of generations, averaged over 50 independent experiments and see which GA yields the best result. The performances of various GAs as a function of number of
Table 1: Statistics of the best chromosomes obtained by various GAs at the end of 200-th generation for $N=80$. Data are collected from 100 independent experiments for each GA variant. The best chromosomes obtained from SGAs do not necessarily satisfy the special structural property, and therefore the numbers of instances of best chromosomes that satisfy the property are indicated. The quantity Prs stands for the Probability that Structural property is observed over 100 independent experiments. In the calculation of the quality measure, $d_{1}(s)$ and $d_{2}(s)$ for the case of SGA, we only count those chromosomes satisfying the special structural property.

\begin{tabular}{|c|c|c|c|}
\hline GA Variant & Prs & $d_{1}(s)$ & $d_{2}(s)$ \\
\hline SGANoX & $97 / 100$ & 3.60825 & 7.87629 \\
\hline SGA & $96 / 100$ & 3.48958 & 6.84375 \\
\hline SGANoXwSPInit & $97 / 100$ & 4.89691 & 8.17526 \\
\hline SGAwSPInit & $100 / 100$ & 4.75 & 6.47 \\
\hline SPGANoX & $100 / 100$ & 0.25 & 0.89 \\
\hline SPGA & $100 / 100$ & 0.00 & 0.54 \\
\hline
\end{tabular}

generation when searching for optimal sequence with length $N=200$ are shown in Fig. 2. We see that SPGAs outperform problem-independent SGAs. In fact, for SGAs with structure-preserving initialization, the average best fitness value drops as the number of generation increases. Let us compare this curious feature of decreasing average fitness of SGAs for large $N(=200)$ with the results for small $N(=80)$ shown in Fig. 1. The optimality gaps are all decreasing with generation number in Fig. 1, implying that the average fitness of all GAs are increasing. We also see that SGAs are able to take advantage of the good quality initial chromosomes resulted from the structuralpreserving initialization. However, when $N$ is large, as shown in Fig. 2 for $N=200$, SGAs no longer have the ability in exploiting the good quality of the initial chromosomes. In Fig. 2, we observe the merging of several curves at large generation number:

1. $\operatorname{SPGA}(\diamond)$ merges with SPGANoX $(\square)$

2. SGAwSPInit (०) merges with $\operatorname{SGA}(\boldsymbol{\square})$

3. $\operatorname{SGANoX}(\bullet)$ merges with $\operatorname{SGANoXwSPInit}(\bullet)$;

We see from 1 that SPGA with crossover operator, does not have noticeable advantage over SPGANoX in terms of best fitness value in the population, suggesting structure-preserving crossover operator does not provide additional advantage for large-size problem. From 2 we see that for SGA, the special initialization with structure preserving features is irrelevant for large $N$. From 3, we see similar effect as 2 without crossover for SGA, when $N$ is large.

In Table 2, we see the average of the best fitness values at the end of 200-th generation and the standard deviation. Not only do SPGAs (SPGANoX and SPGA) achieve higher best fitness value, they 
Table 2: Best fitness values statistics for $N=200$ at the end of the experiment. Data are collected from 50 experiments.

\begin{tabular}{|c|c|c|}
\hline GA Variant & Avg. best fitness & Stand. deviat. \\
\hline SGANoX & 12.1 & 0.231 \\
\hline SGA & 13.4 & 0.250 \\
\hline SGANoXwSPInit & 12.1 & 0.228 \\
\hline SGAwSPInit & 13.3 & 0.273 \\
\hline SPGANoX & 15.01 & 0.0805 \\
\hline SPGA & 15.00 & 0.0978 \\
\hline
\end{tabular}

Table 3: Best fitness values statistics for $N=300$ at the end of the experiment. Data are collected from 50 experiments.

\begin{tabular}{|c|c|c|}
\hline GA Variant & Avg. best fitness & Stand. deviat. \\
\hline SGANoX & 15.9 & 0.364 \\
\hline SGA & 18.0 & 0.360 \\
\hline SGANoXwSPInit & 15.9 & 0.348 \\
\hline SGAwSPInit & 17.7 & 0.334 \\
\hline SPGANoX & 21.90 & 0.0907 \\
\hline SPGA & 21.97 & 0.0893 \\
\hline
\end{tabular}

also have smaller standard deviation in the best fitness value, which means that SPGAs are more reliable. Moreover, SPGANoX and SPGA have similar performance statistically, suggesting structurepreserving crossover operator does not provide noticeable additional advantage for large-size problem.

Results for optimal sequence search with length $N=300$ exhibit similar qualitative features (Table 3). SPGAs outperform SGAs in terms of average best fitness value. SGAs are not able to exploit the good quality chromosomes resulted from the structure-preserving initialization. Similar to the case of $N=200$, the structure-preserving crossover operator does not provide additional benefit in performance.

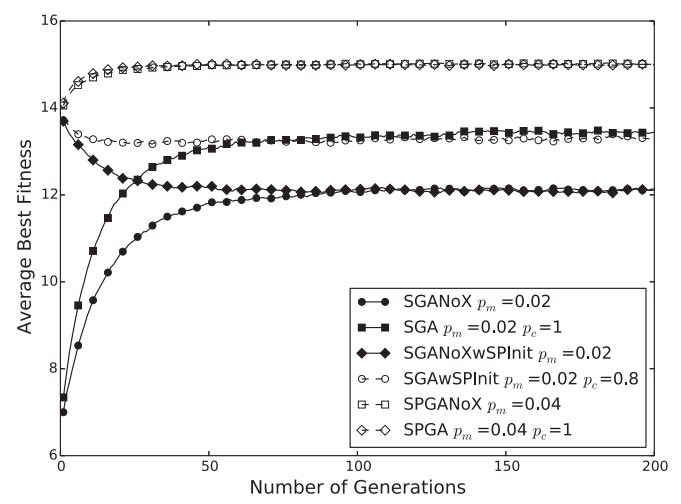

Figure 2: Average best fitness as a function of number of generations for $N=200$. Every curve is an average from 50 independent experiments.

\section{CONCLUSIONS AND FUTURE WORK}

In this paper, we have proposed problem-dependent structure-preserving mutation operator, one-point crossover operator and initialization routine. We have designed several numerical experiments to evaluate the relative efficiency of several GAs incorporating various features deemed important from observation of small $N$ optimal sequences. We have shown that the proposed structure-preserving initialization routine offers high-quality candidate solutions. We have also shown that both structure-preserving mutation operator and one-point crossover operator improves the performance greatly compared to various versions of simple GAs. The various versions of SPGAs are consistently better at figuring out the structure of optimal sequence for medium-size problem, e.g. $N=80$. In medium-size problems, e.g. $N=80$, structurepreserving crossover operator provides noticeable additional advantage; however, for large-size problems, e.g. $\quad N=200$, structure-preserving crossover operator does not provide noticeable additional advantage. This observation could be useful in using GAs to discover the structures of sequences for different lengths. In general, our analysis using GA for Parrondo game sequences can be extended to the analysis of sequences from other fields. For the future works, we could develop a permutation-based evolution operator to further improve the performance of SPGAs.

Within the confine of limited numerical analysis for long sequence, we can conclude that our algorithm is useful in searching for the optimal sequence for Parrondo game. Since the exact solution is not known for long sequence, our method does provide useful candidates for benchmarking. This is important for applications in other sequence analysis for which a good solution, not the mathematically optimal one, is needed. In the context of Parrondo game, Dinis algorithm for short and medium sequences can provide a benchmark for the optimal solution, which we can also obtain by SPGA. However, for very long sequence, when Dinis algorithm is not easily used, our SPGA does provide a good solution. In practical applications in other sequences, we can first analyze the motif in short sequences where exhaustive search provide the optimal solution, and then use those motifs to obtain a good solution for long sequence with SPGA.

\section{ACKNOWLEDGEMENTS}

K. Y. Szeto acknowledges the support of grant FS GRF13SC25and FS GRF14SC28. Degang Wu would 
like to thank Zhangyu Chang for helpful discussions.

\section{REFERENCES}

Allison, A. and Abbott, D. (2002). The physical basis for parrondo's games. Fluctuation and Noise Letters, 2(04):L327L341.

Clote, P. (2005). An efficient algorithm to compute the landscape of locally optimal RNA secondary structures with respect to the nussinov-jacobson energy model. Journal of computational biology, 12(1):83101.

Debenedetti, P. G. and Stillinger, F. H. (2001). Supercooled liquids and the glass transition. Nature, 410(6825):259267.

Ding, Y.-S. and Zhang, T.-L. (2008). Using chous pseudo amino acid composition to predict subcellular localization of apoptosis proteins: An approach with immune genetic algorithm-based ensemble classifier. Pattern Recognition Letters, 29(13):1887-1892.

Dinis, L. (2008). Optimal sequence for parrondo games. Physical Review E, 77(2):021124.

Dinis, L. and Parrondo, J. M. R. (2003). Optimal strategies in collective parrondo games. Europhysics Letters (EPL), 63:319-325.

Dins, L. and Parrondo, J. M. (2004). Inefficiency of voting in parrondo games. Physica A: Statistical Mechanics and its Applications, 343:701-711.

Doye, J. P. (2002). Network topology of a potential energy landscape: A static scale-free network. Physical review letters, 88(23):238701.

Fong, L. Y. and Szeto, K. Y. (2001). Rules extraction in short memory time series using genetic algorithms. The European Physical Journal B-Condensed Matter and Complex Systems, 20(4):569572.

Goldberg, D. E. and Holland, J. H. (1988). Genetic algorithms and machine learning. Machine learning, 3(2):9599.

Harmer, G. P. and Abbott, D. (1999). Game theory: Losing strategies can win by parrondo's paradox. Nature, 402(6764):864864.

Harmer, G. P. and Abbott, D. (2002). A review of parrondos paradox. Fluctuation and Noise Letters, 2(2):R71R107.

Holland, J. H. (1975). Adaptation in natural and artificial systems: An introductory analysis with applications to biology, control, and artificial intelligence. U Michigan Press.

Jiang, R., Szeto, K. Y., Luo, Y. P., and Hu, D. C. (2000). Distributed parallel genetic algorithm with path splitting scheme for the large traveling salesman problems. In Proceedings of Conference on Intelligent Information Processing, 16th World Computer Congress, page 2125.

Messom, C. (2002). Genetic algorithms for auto-tuning mobile robot motion control.

Parrondo, J. M. R. (1996). How to cheat a bad mathematician. EEC HC\&M Network on Complexity and Chaos.
Parrondo, J. M. R., Dinis, L., Garca-Torao, E., and Sotillo, B. (2007). Collective decision making and paradoxical games. The European Physical Journal Special Topics, 143(1):39-46.

Patel, A. N., Davis, D., Guthrie, C. F., Tuk, D., Nguyen, T. T., Williams, J., and others (2005). Optimizing cyclic steam oil production with genetic algorithms. In SPE Western Regional Meeting. Society of Petroleum Engineers.

Pond, S. L. K., Posada, D., Gravenor, M. B., Woelk, C. H., and Frost, S. D. W. (2006). GARD: a genetic algorithm for recombination detection. Bioinformatics, 22(24):3096-3098.

Shiu, K. L. and Szeto, K. Y. (2008). Self-adaptive mutation only genetic algorithm: An application on the optimization of airport capacity utilization. In Intelligent Data Engineering and Automated LearningIDEAL 2008, page 428435. Springer.

Szeto, K. Y. and Fong, L. Y. (2000). How adaptive agents in stock market perform in the presence of random news: A genetic algorithm approach. In Intelligent Data Engineering and Automated LearningIDEAL 2000. Data Mining, Financial Engineering, and Intelligent Agents, page 505510. Springer.

Toral, R., Amengual, P., and Mangioni, S. (2003). Parrondo's games as a discrete ratchet. Physica A: Statistical Mechanics and its Applications, 327(1-2):105110.

Wagon, S. and Velleman, D. (2001). Parrondo's paradox. Mathematica in Education and Research, 9(34):8590.

Wales, D. J., Doye, J. P., Miller, M. A., Mortenson, P. N., and Walsh, T. R. (2000). Energy landscapes: from clusters to biomolecules. Advances in Chemical Physics, 115:1112.

Wales, D. J., Miller, M. A., and Walsh, T. R. (1998). Archetypal energy landscapes. Nature, 394(6695):758760.

Wu, D. and Szeto, K. Y. (2014). Extended parrondo's game and brownian ratchets: Strong and weak parrondo effect. Physical Review E, 89(2):022142.

Xia, C., Guo, P., Shi, T., and Wang, M. (2004). Speed control of brushless DC motor using genetic algorithm based fuzzy controller. In Proceeding of the 2004 International Conference on Intelligent Mechatronics and Automation, Chengdu, China, 3rd edn. A Treatise on Electricity and Magnetism, volume 2, page 6873. 